\title{
Normal and unusual transient events in IRAC images
}

Brian Michael Patten, Joseph L. Hora, Giovanni G. Fazio, Pauline Barmby, Zhong Wang, et al.

Brian Michael Patten, Joseph L. Hora, Giovanni G. Fazio, Pauline Barmby, Zhong Wang, David Makovoz, "Normal and unusual transient events in IRAC images," Proc. SPIE 5487, Optical, Infrared, and Millimeter Space Telescopes, (12 October 2004); doi: 10.1117/12.550543

Event: SPIE Astronomical Telescopes + Instrumentation, 2004, Glasgow, United Kingdom 


\title{
Normal and unusual transient events in IRAC images
}

\author{
Brian M. Patten ${ }^{* a}$, Joseph L. Hora ${ }^{\mathrm{a}}$, Giovanni G. Fazio ${ }^{\mathrm{a}}$, Pauline Barmby ${ }^{\mathrm{a}}$, Zhong Wang ${ }^{\mathrm{a}}$, \\ David Makovoz \\ ${ }^{a}$ Harvard-Smithsonian Center for Astrophysics, 60 Garden St., Cambridge, MA USA 02138; \\ ${ }^{\mathrm{b}}$ Spitzer Science Center, MS 220-6, Caltech, Pasadena, CA, USA 91125
}

\begin{abstract}
The Spitzer Space Telescope Infrared Array Camera (IRAC) is a four-channel camera that uses two pairs of $256 \times 256$ pixel InSb and Si:As IBC detectors to provide simultaneous images at 3.6, 4.5, 5.8, and $8 \mu \mathrm{m}$. IRAC experiences a flux of cosmic rays that produce transient events in images from each of the arrays, with 5-7 pixels per second being affected in an IRAC integration. The vast majority of these transient events can be adequately characterized so they can be effectively detected and flagged by a pipeline software module. However, because of the nature of the arrays and their arrangement in the camera structure, a small fraction of the cosmic ray hits on IRAC produce transients with unusual morphologies that cannot be characterized in a general way. We present nominal cosmic ray rates observed for IRAC on-orbit and rates observed during a period of elevated solar proton flux following a series of X-class solar flares in late 2003. We also present a guide for observers to help identify unusual transient events in their data. We comment on the physical nature of the production of many of these unusual transients and how this mechanism differs from the production of "normal" transient events.
\end{abstract}

Keywords: infrared detectors, cosmic rays, image processing and artifacts

\section{INTRODUCTION}

The Infrared Array Camera (IRAC) $)^{1,2,3,4}$ is one of three focal plane instruments in the Spitzer Space Telescope $e^{5,6}$. IRAC is a four-channel camera that obtains simultaneous images at 3.6, 4.5, 5.8, and $8 \mu \mathrm{m}$. Two adjacent $5.2 \times 5.2$ arcminute fields-of-view in the Spitzer focal plane are viewed by the four channels in pairs (3.6 and $5.8 \mu \mathrm{m} ; 4.5$ and $8 \mu \mathrm{m}$ ). All four detector arrays in the camera are $256 \times 256$ pixels in size, with the 3.6 and $4.5 \mu \mathrm{m}$ channels using InSb arrays and the 4.5 and $8 \mu \mathrm{m}$ channels using Si:As IBC arrays. Both detector types present pixels $30 \mu \mathrm{m} \times 30 \mu \mathrm{m}$ to the incoming light, but the Si:As detectors are thicker $(30 \mu \mathrm{m})$ compared to the InSb detectors $(7 \mu \mathrm{m})$. Figure 1 shows the basic layout of the camera and the relative placement of the four focal plane assemblies (FPAs).

Launched on August 25, 2003, Spitzer was inserted into an Earth-trailing solar orbit, which places the spacecraft well outside any shielding effects of the Earth's magnetosphere or enhancements in charged particle flux due to the Earth's radiation belts ${ }^{7}$. Following launch, Spitzer underwent a 3-month evaluation during the In-Orbit Checkout (IOC) and Science Verification periods, during which the telescope was focused and a functional checkout of the instruments and observational templates was performed. During this time, IRAC was powered on for more than 900 hours, collecting in excess of 150,000 frames of calibration and science data. The camera is now being used regularly to collect science data as a part of the nominal operations phase of the mission.

On orbit, the arrays experience a flux of high energy particles ("cosmic rays"). These cosmic rays consist primarily of solar protons and galactic cosmic rays - mostly protons, but also alpha particles and other heavier nuclei. These cosmic rays produce transient events in IRAC images. Transients are events where an apparent signal is produced in one or more pixels over a time much shorter than the total exposure time for the image. These apparent signals are random both spatially and temporally. While the vast majority of these events can be adequately characterized so they can be detected and flagged by the IRAC pipeline software, a small fraction of these events produce transients with unusual morphologies that cannot be characterized in a general way.

\footnotetext{
*bpatten@cfa.harvard.edu; phone 1617 496-7770; fax 1617 495-7490; cfa-www.harvard.edu
} 


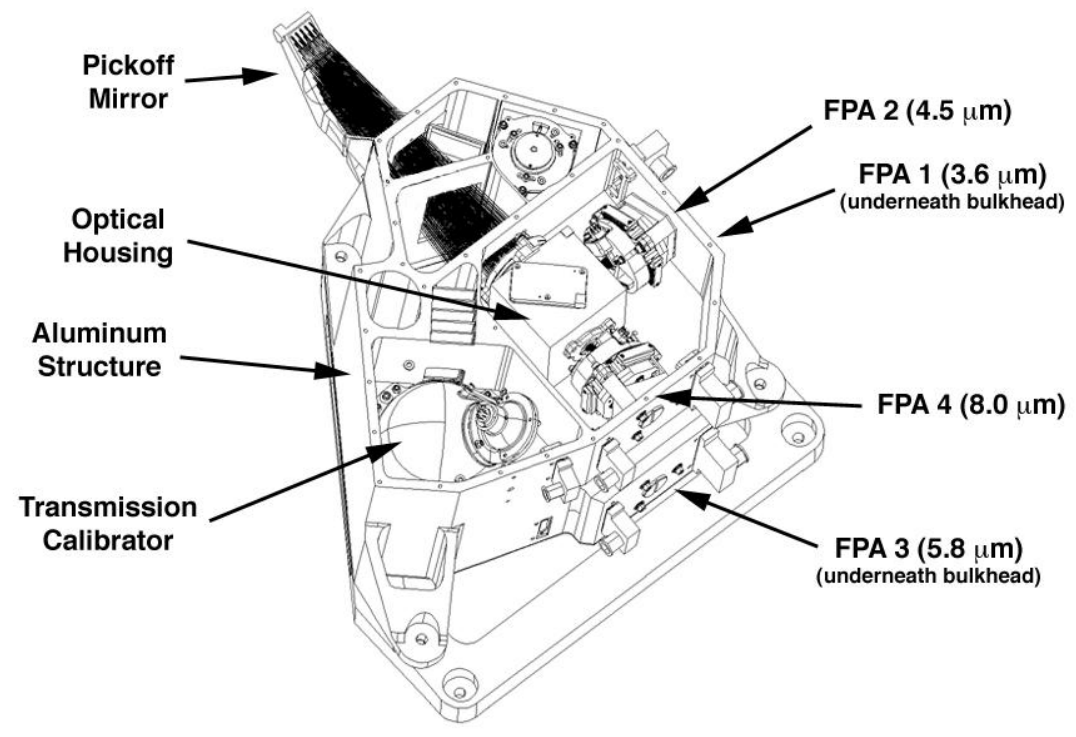

Fig. 1. This drawing shows IRAC with the top cover removed. Light from the telescope reflects off the pickoff mirrors that define two separate fields in the focal plane. The light is then refracted by doublet camera lenses, with the short wavelength light reflected by the beamsplitter and the long wavelength light transmitted. The light for each channel then passes through a filter and enters the array. Shown in this figure are the relative positions of each focal plane assembly (FPA) within the camera body. Visible are FPAs for channels 2 and 4, while in an identical bay underneath the bulkhead are the FPAs for channels 1 and 3 . Within the integrated telescope, the shielding at the location of the IRAC FPAs is $\sim 100 \mathrm{~mm}$ of aluminum ${ }^{8}$, with the two Si:As detectors being the most outboard of the four FPAs.

In this paper we will present cosmic ray rates observed for IRAC through April 2004 as well as during a two-week period of elevated solar proton flux following a series of X-class solar flares starting in late-October 2003 . For the unusual cosmic ray morphologies, we present rates and a guide for observers to recognizing these unusual events in their IRAC data. We also discuss transient mitigation strategies and solutions for Spitzer IRAC observers.

\section{NORMAL TRANSIENT EVENTS}

Data to measure the cosmic ray rates and characteristics were obtained from an IOC program specifically designed for this task as well as from regular calibration data used to characterize the IRAC darks as a part of nominal operations. Both tasks make use of two fields located near the north ecliptic pole (NEP). These fields were selected to be relatively devoid of astronomical sources visible in 100 -second frame times.

Cosmic rays affect approximately 5 pixels per second in channels 1 and $2(3.6$ and $4.5 \mu \mathrm{m})$ and approximately 7 pixels per second in channels 3 and $4(5.8$ and $8.0 \mu \mathrm{m})$ in an IRAC integration. The measured cosmic ray rates from just after Spitzer launch through April 2004 are presented in section 4 of this paper. The most common cosmic rays create transients which are confined to a few pixels around the peak and do not affect the pixel performance in subsequent frames. Typical cosmic ray transients in channels 1 and 2 (the InSb detectors) appear more compact than those seen in the Si:As detectors used for channels 3 and 4. For the InSb detectors, these transients are only a few pixels in size and are close to square in shape. Cosmic rays in the Si:As detectors appear more as streaks or larger blobs of several pixels in size. Cosmic ray transients are also wider in channel 3 compared to channel 4. This is because channel 3 is operated at a lower bias than channel 4, which leads to more charge spreading. During an IOC test, the channel 3 bias voltage was lowered, increasing the bias; while this did result in narrower cosmic ray transients, along with improvements in the QE and PSF width, the improvements observed were not significant enough to warrant changing the bias, since all of the ground calibration of the detector would have to be redone in-flight. Figure 2 shows portions of two 100-second IRAC frames obtained during in one of the dark fields near the NEP during IOC. The stars have been subtracted to show only the cosmic rays in the images. 

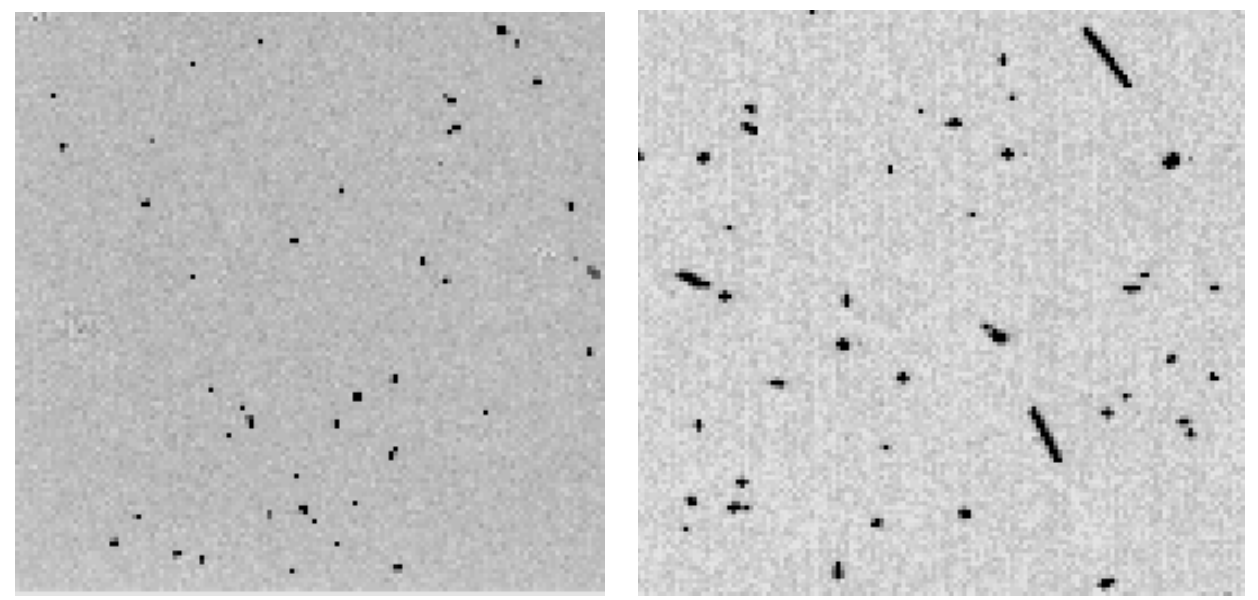

Fig. 2. These images show $140 \times 140$ pixel sections of two 100-second IRAC frames obtained during IOC. The stars have been subtracted to show only cosmic rays in the images. Cosmic rays in channel 1 (left) tend to be more compact than those in channel 3 (right), which can appear as streaks and blobs, depending on the angle of incidence of the cosmic ray as it penetrates the thicker Si:As detectors.

As a part of the IRAC Basic Calibrated Data (BCD) pipeline, transients which result in high intensity small clusters of pixels are flagged by the software. These events are recorded by setting a bit in the data collection event (DCE) status mask (the "dmask"). This dmask is provided to observers as a part of the standard BCD products.

\section{UNUSUAL TRANSIENT EVENTS}

One of the tasks carried out during IOC through the first nominal operations campaign was monitoring of the IRAC dark stability. This task, executed shortly after the instrument was turned on, consisted of a series of 30 -second frames taken in one of the dark fields located near the NEP. A set of five dithered frames, repeated 6 times using the same pattern, was used to determine the stabilization time scale of the dark levels in the first 30 minutes after IRAC was powered on as well as to monitor any changes in the dark pattern between instrument power cycles. In addition, periodic monitoring of the same dark field near the NEP was carried out at various intervals during several of the longer IRAC testing campaigns and during the first nominal operations campaign.

As a part of the analysis procedure, the data from the dark monitoring task were all visually inspected (over 10,000 frames in total). Early in IOC, a number of transient events with unusual morphologies were noticed. These were called "cosmic ray comets" because they appeared to have a fuzzy tail and/or halo instead of the usual sharp-edged streaks. These "comets" only appear in the Si:As detectors. Their morphology appears to depend on the angle of incidence of the cosmic ray.

Additional unusual transient morphologies were noted in subsequent IOC campaigns in all four IRAC channels. Unlike normal transient events, the unusual transients as a class affect tens to hundreds of pixels. They can also trigger detector effects associated with bright sources ${ }^{2,9}$. These include: multiplexer bleed ("muxbleed") - an effect observed in the $\mathrm{InSb}$ detectors where a high signal source forms a trail in the readout direction for a number of consecutive readouts. Because there are four readout amplifiers per array, the trail appears every fourth pixel; column pulldown - a bright source results in a decrease of $20-60 \mathrm{DN}$ in the entire column(s) centered on the source; banding - seen only in the $\mathrm{Si}$ As detectors, the rows and columns containing a bright source are brighter than the background levels; latent images - residual images which remain on the array in subsequent frames. Most latent images decay to the level of the background after several frames. 

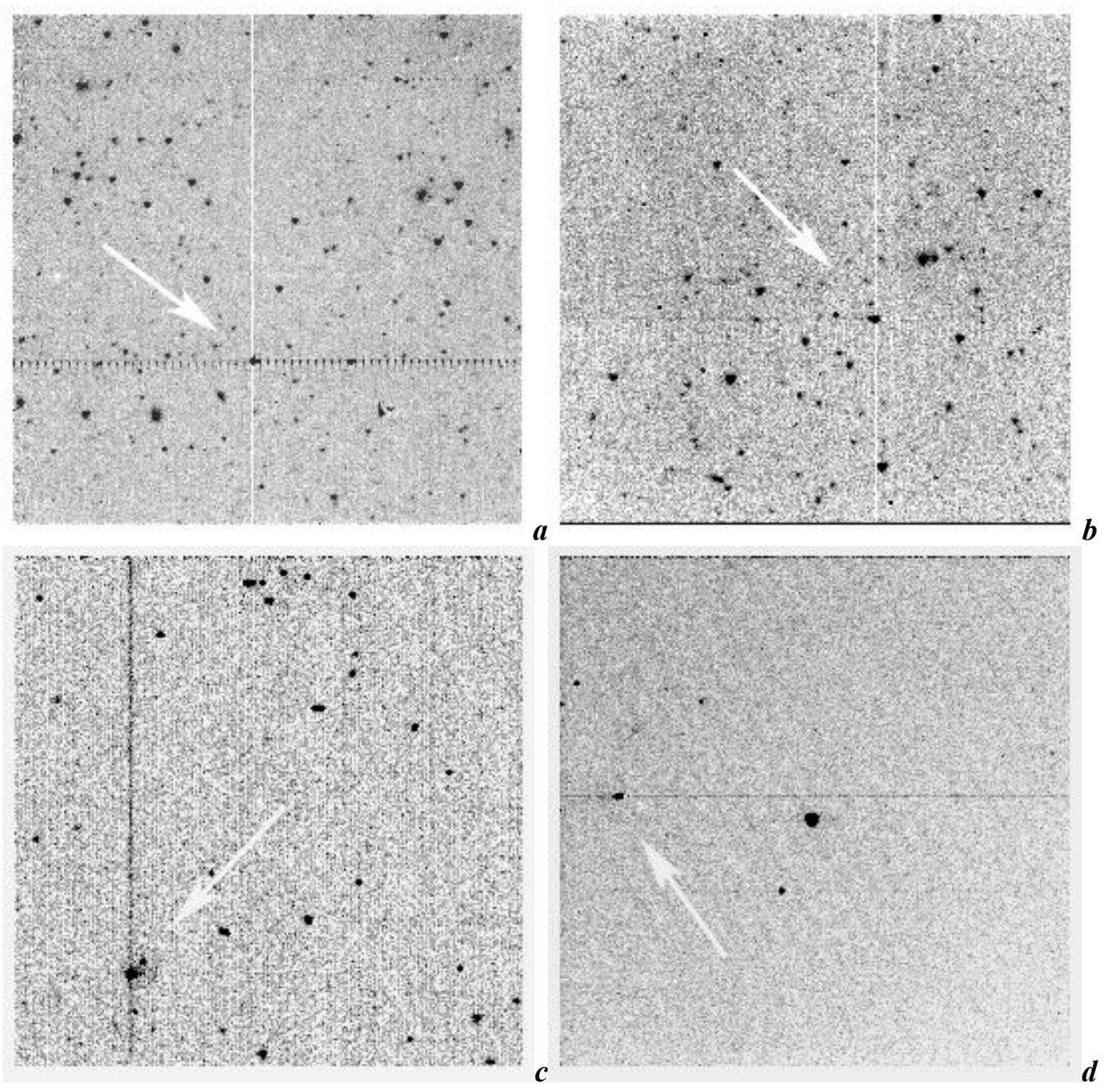

Fig. 3. Examples of strong cosmic ray transients produced in each of the four IRAC channels. For the InSb detectors, channel $1(a)$ and channel $2(b)$, energetic cosmic rays produce a few saturated or high count pixels along with muxbleed (along the rows) and column pulldown (along columns). For the Si:As detectors, channel $3(c)$ and channel $4(d)$, energetic cosmic rays result in transients which tend to saturate or leave high counts in more pixels than channels 1 and 2, as well as banding (either in the horizontal or vertical directions or both).

Most unusual transients fall into one of several groups:

- Strong cosmic ray hit: (Figure 3) Saturates one or more pixels, triggers muxbleed and column pulldown (channels 1 and 2) or banding (channels 3 and 4),

- Scattering: (Figure 4) Low energy ion scatters within the detector leaving a segmented track showing direction changes,

- Particle shower: (Figure 5) High energy ion collision outside the detector or within the detector (all four IRAC channels) produces a shower of secondary particles over the detector. A strong cosmic ray hit is often associated with the shower. These showers can trigger other detector effects such as those listed for strong cosmic ray hits above,

- Cosmic ray comets: (Figure 6) High energy ion produces a fuzzy tail or beam. In some cases the tail is observed, but no associated cosmic ray hit appears to be present at the nominal "head" of the comet.

The production of secondary particle showers - mostly protons and neutrons - results from the passage of primary cosmic rays through the spacecraft and camera structures. Based on pre-launch calculations, the expectation was that less than $2 \%$ of primary cosmic ray protons will produce secondary particle showers ${ }^{10}$. The observed rates of secondary showers observed in the IRAC detectors (given below) are well within this expectation. 

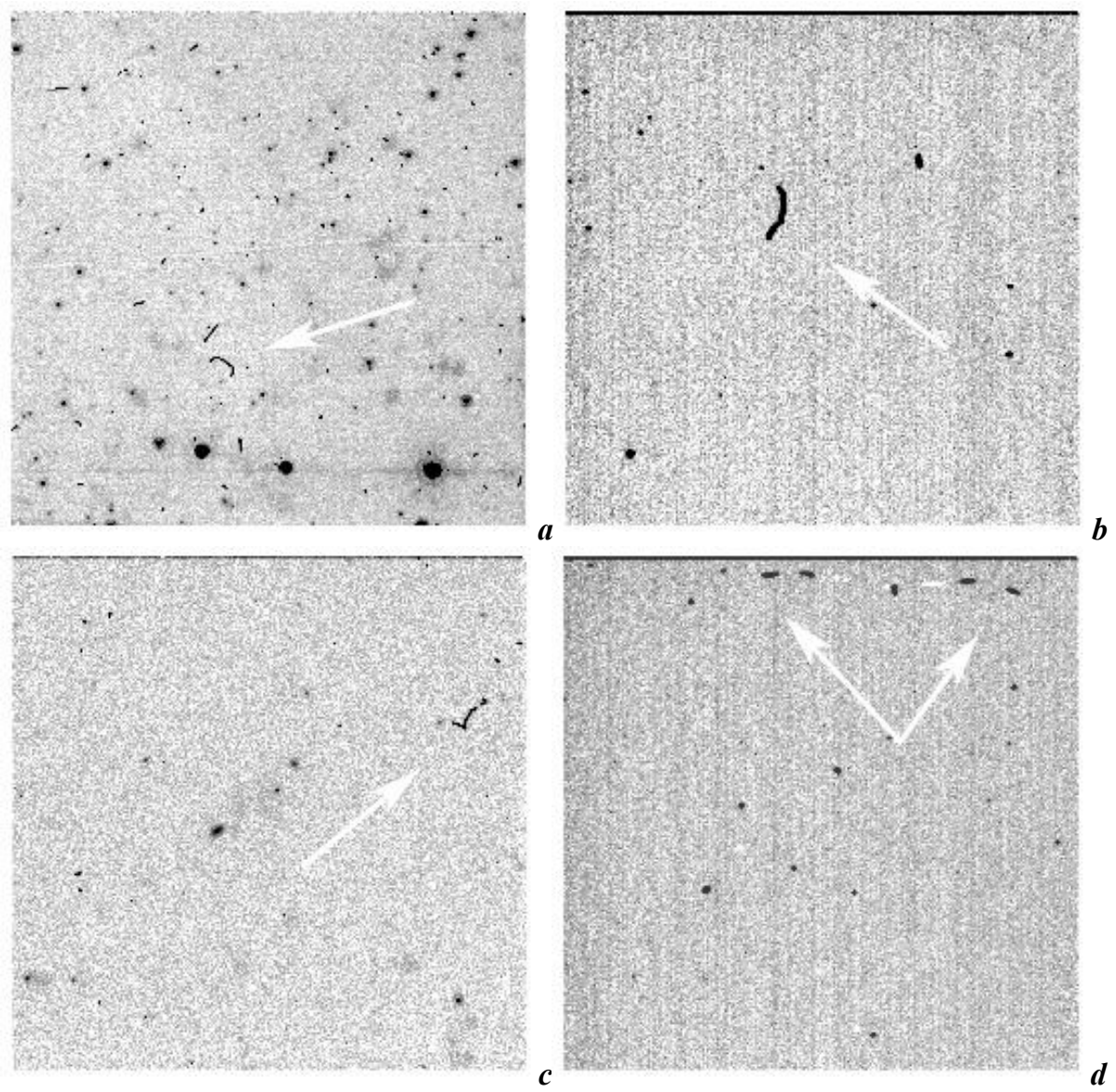

Fig. 4. Examples of lower energy cosmic rays undergoing scattering within the Si:As arrays producing tracks with unusual shapes. (a) Channel 4 U-shaped track resulting from at least two scattering events. (b) Channel 3 apparently curved track due to at least two scattering events. (c) Check-mark shaped track in channel 4. (d) This unusual set of tracks in channel 3 was likely produced by the same cosmic ray as it scattered back and forth in the plane of the detector crossing through different layers of the device.

The effect in the Si:As detectors that allows the cosmic ray comets to be produced is not fully understood at this time. It's likely the passage of a cosmic ray through an electric field free region in the detector, such as the thick $(500 \mu \mathrm{m}) \mathrm{Si}$ substrate layer, produces a cloud of liberated charge carriers which then diffuse until some of them enter a layer with an electric field where they are then driven across the infrared active region where they are collected by the contacts. An analogous effect has been observed for cosmic ray hits in the Chandra ACIS detectors ${ }^{11}$. Some evidence in the test data suggests this particular morphology disappears entirely or is greatly reduced after annealing of the channel 3 and 4 FPAs. That is, heating the arrays above their nominal operating temperatures to remove hot pixels or residual images. At the end of IOC, a test was conducted on annealing strategy in the middle of an IRAC campaign. The success of this test led to regular annealing being implemented as a part of the IRAC power-on sequence. Since this first annealing test, cosmic ray comets have not been observed in subsequent IRAC dark monitoring tests.

For channels 1 and 2 (InSb), the combined mean rate over all campaigns for strong cosmic ray hits is $7.3 \times 10^{-3}$ per second, or about 1 event every 140 seconds. Particle shower events occur at a rate of $2.6 \times 10^{-4}$ per second or about 1 such event per hour. For channels 3 and 4 (Si:As), the combined mean rate of strong cosmic ray hits over all campaigns is $5.0 \times 10^{-3}$ per second or about 1 event every 200 seconds. For cosmic ray comets, the overall combined rate is $3.3 \times 10^{-3}$ per second or about 1 event every 300 seconds. For particle shower events the rate is $5.8 \times 10^{-4}$ per second or about 1 event every 30 minutes. Particle shower events may be more common in the Si:As detectors because the relative thickness of these detectors compared to the InSb arrays may make them more sensitive to a wider range of angles of incidence for these events. 

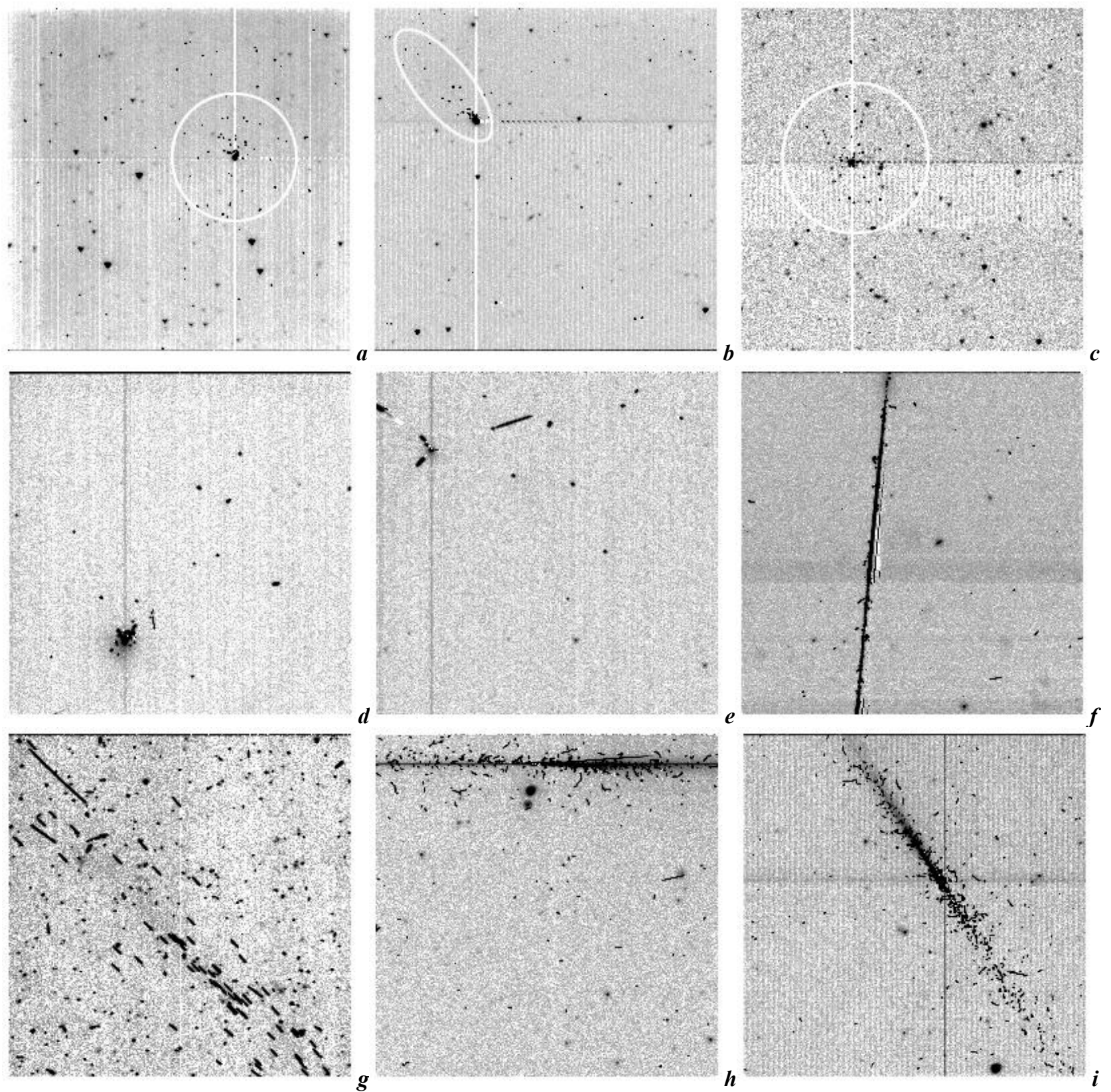

Fig. 5. A variety of cosmic ray hits which produced particle showers recorded by the arrays. (a) Strong cosmic ray hit with secondary particle shower in channel 1 . The area with majority of secondary particle hits is circled. This frame was taken before the telescope was focused, so the stars seen in this image appear as triangles. Also seen are muxbleed and column pulldown due to the transient. (b) and (c) Strong cosmic ray hits with secondary particle showers in channel 2. Also seen are muxbleed and column pulldown. $(d)$ and $(e)$ Strong cosmic ray hits in channel 3 with secondary particle showers. Both of these hits also show banding. (f) In this case a cosmic ray appears to have passed through channel 4 in the plane of the array creating a bright track but also having collisions which made several short secondary particle tracks along the main trail. ( $g$ ) This channel 3 secondary particle shower probably originated from a cosmic ray hit outside the array itself. $(h)$ and $(i)$ These channel 4 cosmic rays hits seem to have created secondary particle showers which intersected the array in a plane. 

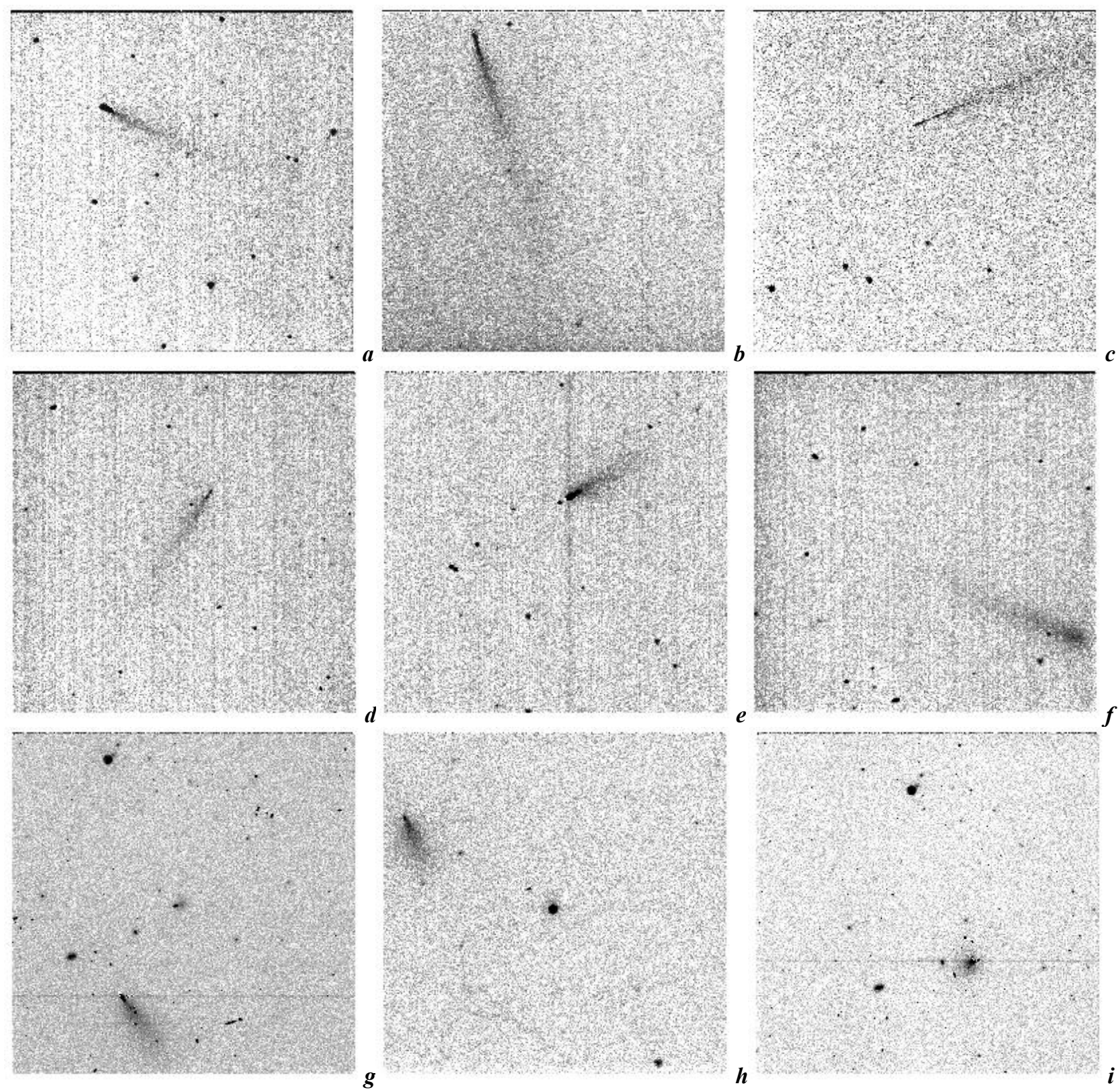

Fig. 6. Examples of "cosmic ray comets" seen in IRAC channels $3(a)-(f)$ and $4(g)-(i)$. Most of these unusual transients have a distinctive cosmic ray hit in the area of highest intensity, however some (like $(f)$ for example) appear as a fuzzy tail without a hit near the "head" of the comet. The length of the tail is likely related to the angle of incidence of the cosmic ray that creates the transient. While the tails can affect tens to hundreds of pixels and create latent images in subsequent frames, they are typically only a few DN above the background.

The outboard position in the telescope and the intrinsic properties of Si:As detectors as compared to the InSb detectors may account for the higher overall cosmic ray rates seen in IRAC channels 3 and 4 . However, for all unusual transient events taken together for both array types, we find for the InSb arrays about 1 event every 140 seconds, while for the $\mathrm{Si}$ As arrays the combined rate is about 1 event every 120 seconds. The fact that these two rates are not significantly different from one another suggests that the InSb and Si:As arrays are seeing the same basic types of energetic particle hits. The energetic cosmic ray hits in the Si:As arrays simply manifest themselves with a wider range of morphologies. 


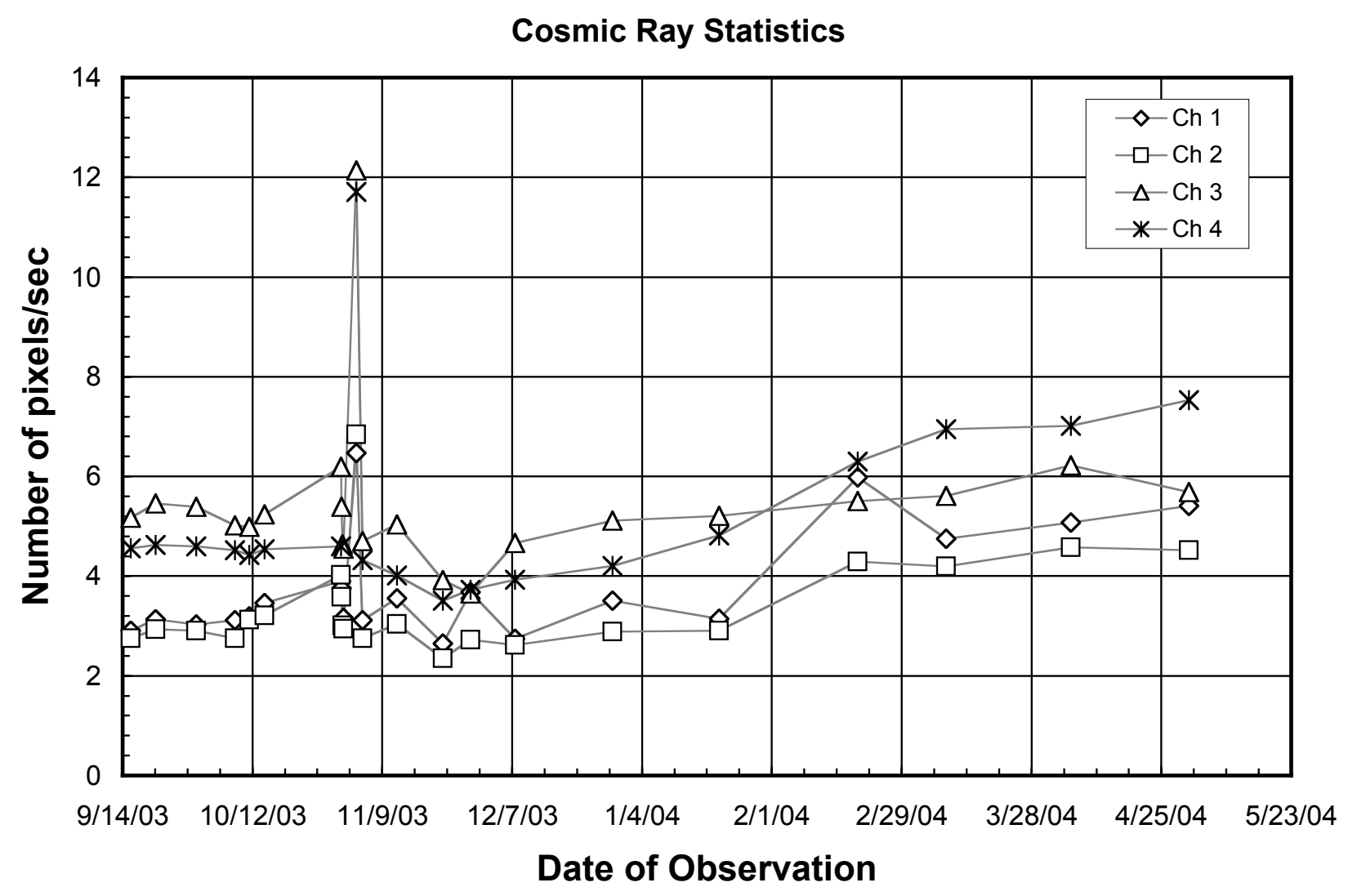

Fig. 7. Total cosmic ray rates for each IRAC channel for all campaigns from IOC just after launch through April 2004. The elevated rates during a relatively quiescent period following a series of X-class solar flares in late October 2003 are seen as the sharp peak in this plot. During the flares, IRAC was powered off.

\section{THE EFFECTS OF THE X-CLASS SOLAR FLARE EVENTS OF LATE 2003}

Starting on October 28, 2003, a series of eleven X-class solar flares (the most powerful class) were observed over the next 14 days, with the largest solar flare ever recorded (>X-28) occurring on November 04, 2003. The Spitzer mission flight rules prohibit operation of the observatory when the flux of $>100 \mathrm{MeV}$ solar protons is above 100 PFU (where a "proton flux unit" $=1$ proton $/ \mathrm{cm}^{2} / \mathrm{sec} / \mathrm{sr}$ ). The observatory was allowed to idle with all instruments off for about 2 days after the October 28 event.

IRAC was last powered on about a week before the October 28 flare, when the solar proton levels were about 0.02 PFU. IRAC was already powered off when the highest proton flux from the flares on October 28 occurred. IRAC was powered-on for a pair short campaigns on October 31 and November 03 designed to evaluate the instrument performance and to anneal the detectors to fix radiation-damaged pixels. During these flare recovery campaigns, the solar proton levels were within operating limits but were above normal at values of $0.2-3$ PFU. The next "nominal" IOC campaign began late on November 04, just before the large solar flare of that date. Because this flare was mainly directed away from the Earth and Spitzer, no major effects were seen. 

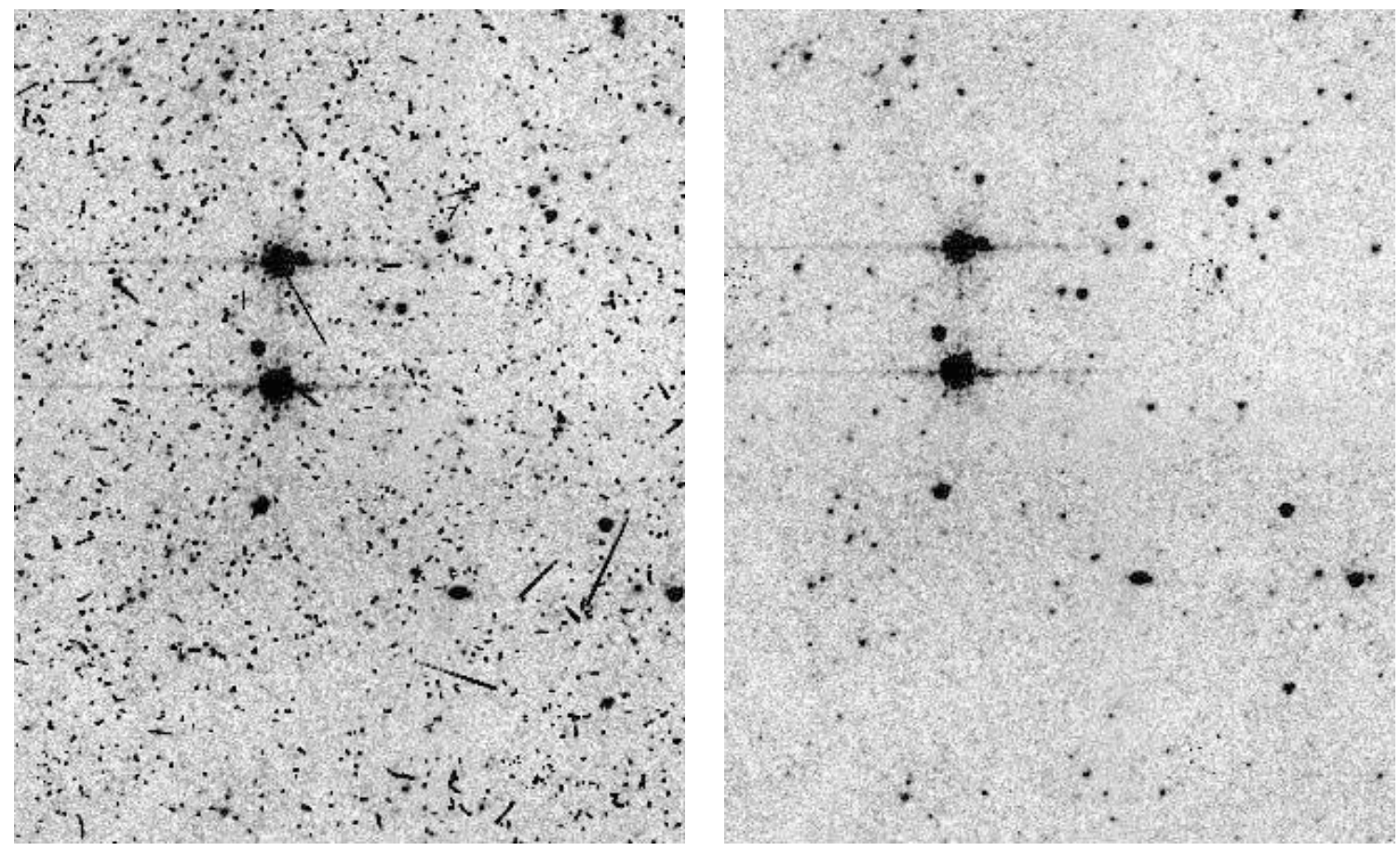

Fig. 8. Before (left) and after (right) images of an IRAC channel 4 mosaic for data with a depth coverage of 16 individual exposures showing the ability of the SSC mosaicer to clean final mosaic of transient events using standard multi-frame outlier rejection. The images shown are $375 \times 316$ IRAC pixels in dimension.

During the flare recovery campaigns, the total cosmic ray rates were about a factor of two higher than in the pre-flare operations period (Figure 7). It was not possible to evaluate the rate of unusual transients since only a limited set of tasks were run in the very short flare recovery campaigns. In the nominal IOC campaign that began on November 04 , there was no convincing evidence in the dark stability monitoring data of any elevated rates in unusual transient events.

The main effects of the solar flare on IRAC was an increase in the number of isolated hot pixels $-9,6,0$, and 50 new hot pixels in IRAC channels $1-4$ respectively compared to $35,18,0$, and 10 total hot pixels before the flares. After several subsequent anneals and power cycles all channels appear to have recovered from the increase in hot pixels except for channel 4, which seems to have 4 times as many hot pixels as before the flares. These channel 4 hot pixels may represent permanent damage to the detector. For all IRAC channels, no changes were seen to the number of dead or isolated low response pixels. No change was seen to IRAC sensitivity in any channel. We found that at rates of $>1$ PFU the increased cosmic ray rate was significant on the IRAC detectors and we conclude that we would not be able to perform science observations at rates above 2 PFU even if it was deemed safe to run IRAC from an operational standpoint.

\section{TRANSIENT MITIGATION STRATEGY}

The best strategy to mitigate transient event effects in IRAC images is to use sufficient redundancy in the number of exposures made in the area of the target of interest. That is, when planning their astronomical observation request (AOR), observers should ensure that multiple exposures are made at the same sky coordinates, either by repeated exposures or by dithering the telescope around the target position, or a combination of both methods, in order to allow for the removal of transient events in later processing. If using IRAC in a mapping mode, the observer can, in addition to the other strategies, plan the AOR such that sufficient overlap is made with adjacent map positions to have redundancy in sky coordinates. 

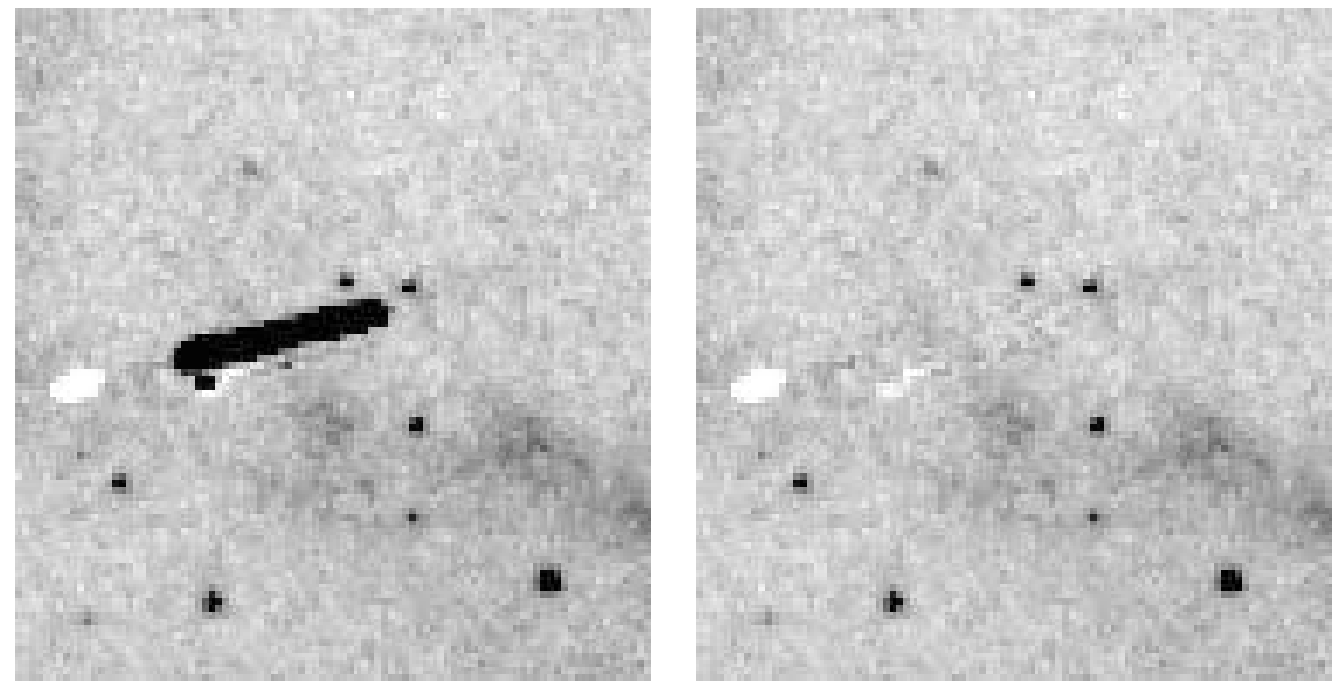

Fig. 9. Example showing the effectiveness of the SSC mosaicer on removing a cosmic ray from IRAC channel 3 data with just a depth coverage of 2 images. The images shown are $69 \times 72$ IRAC pixels, before (left) and after (right) the correction.

The Spitzer Science Center (SSC) mosaicer software ${ }^{12}$ is designed to perform effective detection and masking of the transient events, a.k.a. outlier detection, where outliers are defined as moving objects and cosmic ray hits in the input images. The mosaicer performs outlier detection in interpolated images projected to a common grid. Two algorithms are implemented in the mosaicer which take advantage of the redundant information in the overlap areas of the individual frames. The first - multi-frame temporal outlier detection - is best used when there are many input images available for a particular area (Figure 8). At each grid position n-sigma clipping is done for the stack of individual input pixels for that position in the time domain. The second approach - dual outlier detection - has been designed to deal with areas of shallow coverage in terms of individual images (Figure 9). Here two-stage filtering is performed. The first stage is spatial filtering, whereupon spatial objects - clusters of pixels above a user-specified threshold - are detected in each frame. In the second stage the spatial objects in the interpolated images are matched in the time domain. The unmatched objects are declared outliers and are excluded from the final mosaic. While the SSC automated pipeline doesn't perform dual outlier detection as a part of producing the standard pipeline data products, this algorithm is included in the offline version of the mosaicer software (called mopex) and can be exploited by users, especially for observations with shallow redundancy. The results of the multiframe temporal and dual outlier rejection algorithms are recorded in the "rmask" file produced by the software, which also contains information about single frame outlier detection copied from the dmask.

\section{ACKNOWLEDGEMENTS}

The authors would like to thank Rick Arendt, Jason Surace, and Bill Glaccum (Spitzer Science Center) for additional contributions to this paper. This work is based on observations made with the Spitzer Space Telescope, which is operated by the Jet Propulsion Laboratory, California Institute of Technology under NASA contract 1407. Support for the IRAC instrument was provided by NASA under contract number 960541 issued by JPL.

\section{REFERENCES}

1. Fazio, G. G. et al. 2004. "The Infrared Array Camera (IRAC) for the Spitzer Space Telescope", ApJS Special Spitzer issue

2. Hora, J. L. et al. 2004. "In-flight performance of the Infrared Array Camera (IRAC) for the Spitzer Space Telescope", SPIE, this conference 
3. IRAC - Infrared Array Camera on the Spitzer Space Telescope (http://cfa-www.harvard.edu/irac)

4. Spitzer Science Center, Observatory \& Science Instruments: IRAC (http://ssc.spitzer.caltech.edu/irac)

5. Werner, M. W. et al. 2004. "The Spitzer Space Telescope", ApJS Special Spitzer issue

6. Roellig, T. L. et al. 2004. "On-orbit performance of the Spitzer Space Telescope", SPIE, this conference

7. Garcia, M. D. 2004, "SIRTF Takes Flight", Paper AAS 04-225, Maui, Hawaii, Feb 8-12, 2004

8. Romano, E. 1996. "Radiation Transport Through the SIRTF Observatory for the External Environment to the Focal Plane", IRAC/SDT96-802

9. Hora, J. L. et al. 2000. "Calibration and performance of the Infrared Array Camera (IRAC)", SPIE 4131, 13

10. Kong, R. 1998. "Secondary Particle Radiation Analysis", IRAC/TM98-6001

11. Plucinsky, P. 2004, personal communication.

12. Spitzer Mosaicer User's Guide (http://ssc.spitzer.caltech.edu/postbcd/doc/mosaicer.pdf) 